International Journal of Applied Mathematics

Volume 33 No. $4 \quad 2020,591-597$

ISSN: 1311-1728 (printed version); ISSN: 1314-8060 (on-line version)

doi: http://dx.doi.org/10.12732/ijam.v33i4.4

\title{
RAINBOW CONNECTION NUMBER OF FLOWER SNARK GRAPH
}

\author{
K. Srinivasa Rao ${ }^{1 \S}$ \\ U Vijaya Chandra Kumar ${ }^{2}$, Anjaneyulu Mekala ${ }^{3}$ \\ ${ }^{1}$ Department of Mathematics \\ Sri Venkateshwara College of Engineering \\ Bengaluru 562 157, INDIA \\ ${ }^{2}$ School of Applied Science (Mathematics) \\ REVA University, Bengaluru, INDIA \\ ${ }^{3}$ Gurunanak Institutions Technical Campus \\ Ibrahimpatnam, Hyderabad, INDIA
}

\begin{abstract}
Let $G$ be a nontrivial connected graph on which is defined a coloring $c: E(G) \rightarrow\{1,2, \cdots, k\}, k \in N$ of the edges of $G$, where adjacent edges may be colored the same. A path in $G$ is called a rainbow path if no two edges of it are colored the same. $G$ is rainbow connected if $G$ contains a rainbow $u-v$ path for every two vertices $u$ and $v$ in it. The minimum $k$ for which there exists such a $k$-edge coloring is called the rainbow connection number of $G$, denoted by $\operatorname{rc}(G)$.

In this paper we find the rainbow connection number of flower snark graph and their criticalness with respect to rainbow coloring.
\end{abstract}

AMS Subject Classification: 05C15

Key Words: diameter; edge-coloring; rainbow path; rainbow connection number; criticality

\section{Introduction}

We use [1] for terminology and notation not defined here and consider finite

Received: February 25, 2020

(c) 2020 Academic Publications

$\S_{\text {Correspondence author }}$ 
and simple graphs only.

Let $G$ be a nontrivial connected graph with an edge coloring $c: E(G) \rightarrow$ $\{1,2, \cdots, k\}, k \in N$, where adjacent edges may be colored the same. An edgecolored graph $G$ is called rainbow-connected if any two vertices are connected by a path whose edges have different colors. The minimum $k$ for which there exist a rainbow $k$-coloring of $G$ is called the rainbow connection number of $G$, denoted by $\operatorname{rc}(G)$.

The concept of rainbow connection in graphs was introduced by Chartrand et al. in [2]. In [2] the authors determined $r c(G)$ and $\operatorname{src}(G)$ of the cycle, path, tree and wheel graphs. In [8] and [9] Srinivasa Rao and Murali, studied rainbow connection number $\operatorname{rc}(G)$ and the strong rainbow connection number $\operatorname{src}(G)$ of some classes of graphs like the stacked book graph, the grid graph, the prism graph etc. and discussed the critical property of these graphs with respect to rainbow coloring. The rainbow connection number has been studied for brick product graphs in [10] and [11]. Other results on the rainbow connection number of a graph can be found in [12]. In [6], Nabila et al. determined the rainbow connection number of Origami graphs and Pizza graphs. An overview about rainbow connection number can be found in a book of $\mathrm{Li}$ and $\mathrm{Sun}$ in [4] and a survey by Li et al. in [5].

The flower snarks (for odd $n$ ) form an infinite family of snarks introduced by Isaacs [3]. In this paper we determine the rainbow connection number $r c(G)$ of flower snark graphs and also critical property of $G$ with respect to rainbow connection number.

\section{Definitions}

Definition 1. The flower snarks are a connected, bridgeless cubic graphs. The flower snarks are non-Hamiltonian and non-planar. The flower snark $J_{n}$ can be constructed with the following process:

- Build $n$ copies of the star $K_{1,3}$. Denote the central vertex of each star by $b_{i}$ and the outer vertices $a_{i}, c_{i}$ and $d_{i}$. This results in a disconnected graph on $4 n$ vertices with $3 n$ edges $\left(b_{i} a_{i}, b_{i} c_{i}\right.$ and $b_{i} d_{i}$ for $\left.1 \leq i \leq n\right)$.

- Construct the $n$-cycle $\left(a_{1}, a_{2}, \ldots, a_{n} a_{1}\right)$. This adds $n$ edges.

- Finally construct the $2 n$-cycle $\left(c_{1} c_{2}, \cdots, c_{n} d_{1} d_{2}, \cdots, d_{n} c_{1}\right)$. This adds $2 n$ edges.

The flower snark graph $J_{5}$ and $J_{7}$ are shown in Figure 1. 

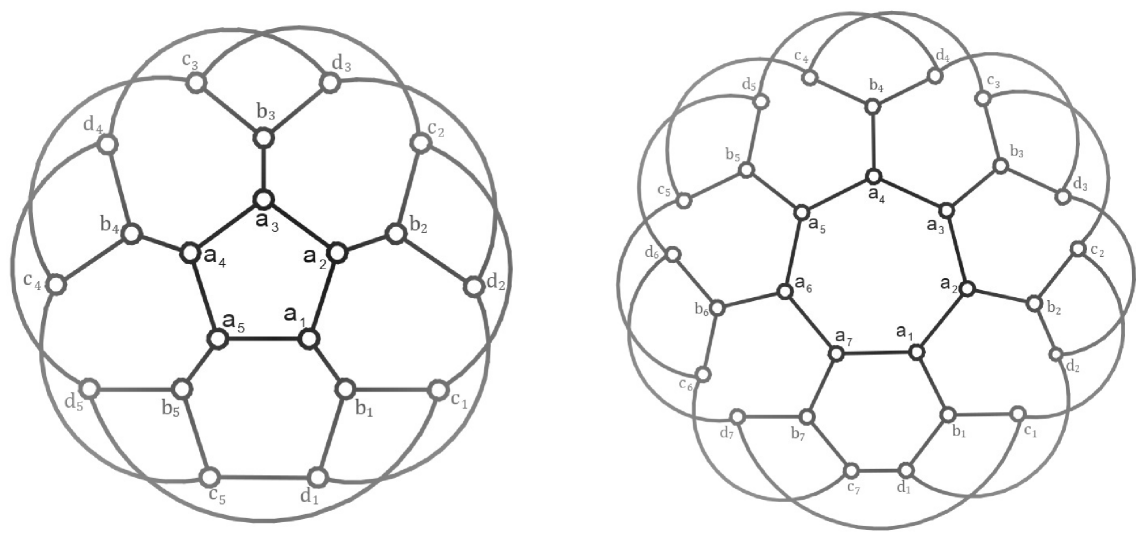

Figure 1: Flower snark graph $J_{5}$ and $J_{7}$

The rainbow criticalness was first studied by Srinivasa Rao and Murali in [8].

Definition 2. A graph $G$ is said to be rainbow critical if the removal of any edge from $G$ increases the rainbow connection number of $G$, i.e. if $\operatorname{rc}(G)=k$ for some positive integer $k$, then $r c(G-e)>k$ for any edge $e$ in $G$.

Theorem 3. Let $G$ be a connected graph on $n \geq 3$ vertices. Then, $r c(G) \geq \max \left\{\operatorname{diam}(G), n_{i}(G)\right\}$, where $n_{i}(G)$ denotes the number of vertices of $G$ which have degree $i$ for $1 \leq i \leq n-1$.

In the next section, we determine rainbow connection number $r c(G)$ for the flower snark graph $J_{n}$ for $n \geq 5$ and odd.

\section{Main results}

Theorem 4. Let $G=J_{n}$. Then for odd $n \geq 5$,

$$
r c(G)=\left\lceil\frac{n}{2}\right\rceil+3 .
$$

Proof. Let us define the vertex set $V$ and the edge set $E$ of $G$ as $V(G)=$ $\left\{a_{1}, a_{2}, \cdots, a_{n}\right\} \cup\left\{b_{1}, b_{2}, \cdots, b_{n}\right\} \cup\left\{c_{1}, c_{2}, \cdots, c_{n}\right\} \cup\left\{d_{1}, d_{2}, \cdots, d_{n}\right\}$, where $b_{i}$ denotes the central vertex of star graph and $a_{i}, c_{i}$ and $d_{i}$ denotes outer vertices and $E(G)=\left\{e_{i}\right\} \cup\left\{a_{i} b_{i}\right\} \cup\left\{b_{i} c_{i}\right\} \cup\left\{b_{i} d_{i}\right\} \cup\left\{e_{i}^{\prime}\right\} \cup\left\{e_{i}^{\prime \prime}\right\} \cup\left\{c_{n} d_{1}\right\} \cup\left\{d_{n} c_{1}\right\}$ where 
$e_{i}$ is the edge $a_{i} a_{i+1}, e_{i}^{\prime}$ is the edge $c_{i} c_{i+1}$ and $e_{i}^{\prime \prime}$ is the edge $d_{i} d_{i+1}$, where $1 \leq i \leq n-1$.

Since $\operatorname{diam}(G)=\left\lceil\frac{n}{2}\right\rceil+1$, it follows that $r c(G) \geq\left\lceil\frac{n}{2}\right\rceil+1$. By the definition of snark graph, $G$ contains $n$ copies of star graph $K_{1,3}$ with the vertices $a_{i}$, $b_{i}, c_{i}$ and $d_{i}$ where $1 \leq i \leq n$. Vertices $a_{i}$ forms inner cycle and from [2] $r c\left(C_{n}\right)=\left\lceil\frac{n}{2}\right\rceil$ so we have to assign $\left\lceil\frac{n}{2}\right\rceil$ colors to the edges of inner cycle. Since $K_{1,3}$ is a subgraph of $G$, again by the definition we have to assign three different colors to the edges other than $\left\lceil\frac{n}{2}\right\rceil$ colors already assigned to the edges of inner cycle. Contrary, let us assign any one of $\left\lceil\frac{n}{2}\right\rceil$ color to the edges $a_{i} b_{i}$, then we fail to get rainbow path between the vertices $a_{\frac{n+3}{2}} b_{\frac{n+1}{2}}, \forall n$ and this is true for other pair of vertices also. So assign $\left\lceil\frac{n}{2}\right\rceil+1$ color to $a_{i} b_{i}$. Similarly, for the edges $b_{i} c_{i}$ and $b_{i} d_{i}$ we should assign two different colors other than $\left\lceil\frac{n}{2}\right\rceil+1$ colors. If not, this coloring will not give rainbow $c_{\frac{n+1}{2}} d_{n}$ path $\forall n$.

Combining all combinations of assignment of colors discussed above and also from Theorem 3,

$$
r c(G) \geq \operatorname{diam}(G)+2, \text { i.e. } r c(G) \geq\left\lceil\frac{n}{2}\right\rceil+3 .
$$

To show $r c(G) \leq\left\lceil\frac{n}{2}\right\rceil+3$, we define a coloring $c: E(G) \rightarrow\left\{1,2, \cdots,\left\lceil\frac{n}{2}\right\rceil+3\right\}$ and assign the colors to the edges of $G$ as,

$$
\begin{aligned}
& c\left(e_{i}\right)= \begin{cases}i & \text { if } \quad 1 \leq i \leq\left\lceil\frac{n}{2}\right\rceil \\
i-\left\lceil\frac{n}{2}\right\rceil & \text { if } \quad\left\lceil\frac{n}{2}\right\rceil+1 \leq i \leq n\end{cases} \\
& c\left(a_{i} b_{i}\right)=\left\lceil\frac{n}{2}\right\rceil+1 \text { for } 1 \leq i \leq n \\
& c\left(b_{i} c_{i}\right)=\left\lceil\frac{n}{2}\right\rceil+2 \text { for } 1 \leq i \leq n \\
& c\left(b_{i} d_{i}\right)=\left\lceil\frac{n}{2}\right\rceil+3 \text { for } 1 \leq i \leq n \\
& c\left(e_{i}^{\prime}\right)=c\left(e_{i}^{\prime \prime}\right)= \begin{cases}i & \text { if } \quad 1 \leq i \leq\left\lceil\frac{n}{2}\right\rceil \\
i-\left\lceil\frac{n}{2}\right\rceil & \text { if }\left\lceil\frac{n}{2}\right\rceil+1 \leq i \leq n-1\end{cases} \\
& c\left(c_{n} d_{1}\right)=c\left(d_{n} c_{1}\right)=n-\left\lceil\frac{n}{2}\right\rceil .
\end{aligned}
$$

From the above assignment, it is clear that for every two distinct vertices $x, y \in V(G)$, there exists an $x-y$ rainbow path.

Hence $r c(G) \leq\left\lceil\frac{n}{2}\right\rceil+3$.

This proves $r c(G)=\left\lceil\frac{n}{2}\right\rceil+3$.

The critical nature of the flower snark graph in Theorem 4 has been observed. This is illustrated in our next result. 
Theorem 5. Let $G=J_{n}$, for odd $n \geq 5$. Then $G$ is rainbow critical, i.e.

$$
r c(G-e)=2 n \text { for } n \geq 5 \text { and odd. }
$$

Proof. We consider vertex set $V(G)$ and edge set $E(G)$ as defined in Theorem 4 . Let $E_{1}, E_{2}$ and $E_{3}$ are the subset of $E(G)$, i.e.,

$$
E(G)=E_{1} \cup E_{2} \cup E_{3},
$$

where

$$
\begin{gathered}
E_{1}=\left\{a_{i} a_{i+1}\right\}-\text { edges of inner cycle, } \\
E_{2}=\left\{a_{i} b_{i}, b_{i} c_{i} b_{i} d_{i}\right\}-\text { edges of star } K_{1,3} \text { and } \\
E_{3}=\left\{c_{i} c_{i+1}, d_{i} d_{i+1}, c_{n} d_{1}, d_{n} c_{1}\right\}-\text { edges of outer cycle. }
\end{gathered}
$$

Consider the graph $G$ and let $e \in E(G)$ be the any edge in $G$. Deletion of the edge $e$ in $G$ does not yield a rainbow path between the end vertices of $e$ in $G-e$. So, we prove this result in following cases.

\section{Case 1:}

Let $e \in E_{1}$. Deletion of edge $e$ in $G$, leads to path of length $n-1$ and for any arbitrary edge $e \in E_{1}$, it requires $n$ colors such that $G-e$ is rainbow connected.

\section{Case 2:}

Now consider the edge $e \in E_{2}$. According to the assignment of colors to the edges of star $K_{1,3}$ in the Theorem $4,\left\lceil\frac{n}{2}\right\rceil+3$ colors is not sufficient in $G-e$. i.e deletion of $e$ will not get rainbow path between the end vertices. So assign same $n$ colors already assigned to the edges of $E_{1}$ in different combination.

Particularly, if $e=b_{i} c_{i}$ or $b_{i} d_{i}$ if we assign same $n$ colors in any combination, the graph $G-e$ will not get rainbow path between the vertices $a_{i}$ to $c_{i}$ or between the vertices $a_{i}$ to $d_{i}$. Also the vertex sets $\left\{a_{i}, a_{i+1}, b_{i}, b_{i+1}, c_{i}, c_{i+1}\right\}$ and $\left\{a_{i}, a_{i+1}, b_{i}, b_{i+1}, d_{i}, d_{i+1}\right\}$ form a cycle of length 6 . Deletion of any edge in the cycle leads to the path of length 5 , which will be the shortest path. It holds up to $n$ vertices. So to get rainbow path in $G-e$ where $e=b_{i} c_{i}$ or $e=b_{i} d_{i}$, it requires different $n$ colors other than the colors already assigned to the edges $a_{i} a_{i+1}$ and $a_{i} b_{i}$.

\section{Case 3:}

Finally let $e \in E_{3}$. In this case also we fail to get rainbow path between the end vertices in $G-e$. Since the edges of $E_{3}$ forms outer cycle and also we show that in above 2 cases, $2 n$ colors requires to get rainbow path in $G-e$, implies assignment of same colors to the edges in different combinations leads $G-e$ is rainbow connected. 
Considering the above 3 cases, it is clear that $r c(G-e) \geq 2 n$.

To prove $r c(G-e) \leq 2 n$, we construct a rainbow coloring $c: E(G) \rightarrow$ $\{1,2, \cdots, 2 n\}$ to the edges of $G$ as,

$$
\begin{gathered}
c\left(a_{i} a_{i+1}\right)=i \text { if } 1 \leq i \leq n \\
c\left(a_{i} b_{i}\right)= \begin{cases}i+\left(\frac{n-1}{2}\right) & \text { if } 1 \leq i \leq\left\lceil\frac{n}{2}\right\rceil \\
i-\left(\frac{n+1}{2}\right) & \text { if }\left\lceil\frac{n}{2}\right\rceil+1 \leq i \leq n\end{cases} \\
c\left(b_{i} c_{i}\right)=c\left(b_{i} d_{i}\right)=i+n \text { if } 1 \leq i \leq n-1 \\
c\left(c_{i} c_{i+1}\right)=c\left(d_{i} d_{i+1}\right)=i+1 \text { if } 1 \leq i \leq n-1 \\
c\left(c_{n} d_{1}\right)=c\left(d_{n} c_{1}\right)=1 .
\end{gathered}
$$

From the above assignment, it is clear that for any two vertices $x, y \in V(G)$, there exists a rainbow $x-y$ path in $G-e$ and hence $r c(G-e) \leq n$.

This proves $r c(G-e)=2 n$.

Hence the proof is done.

\section{Conclusion}

In this paper, we have determined the rainbow connection number of flower snark graph $J_{n}$. The critical property of flower snark graphs with respect to rainbow coloring is also investigated.

Acknowledgement: The first author is thankful to the management and the staff of the Department of Mathematics, Sri Venkateshwara College of Engineering, Bangalore, for their support and encouragement. The authors are also thankful to the management and R \& D Center, Department of Mathematics, Reva University Bangalore.

\section{References}

[1] J.A. Bondy, U.S.R. Murty, Graph Theory, Springer (2008).

[2] G. Chartrand, G.L. Johns, K.A. McKeon, P. Zhang, Rainbow connection in graphs, Mathematica Bohemica, 133, No 1 (2008), 85-98.

[3] R. Isaacs, Infinite families of nontrivial trivalent graphs which are not Tait colorable, Amer. Math. Monthly, 82 (1975), 221239. 
[4] X. Li, Y. Shi, Y. Sun, Rainbow connection of graphs: A survey, Graphs Combin., 29, No 1 (2013), 1-38.

[5] X. Li, Y. Sun, Rainbow Connection of Graphs, Springer-Verlag, New York (2012).

[6] S. Nabila, A.N.M. Salmal, The rainbow connection number of Origami graphs and Pizza graphs, Procedia Computer Science, 74 (2015), 162-167.

[7] I. Schiermeyer, Bounds for the rainbow connection number of graphs, Discussiones Mathematicae Graph Theory, 31, No 2 (2011), 387-395.

[8] K. Srinivasa Rao, R. Murali, Rainbow critical graphs, Int. J. of Comp. Application, 4, No 4 (2014), 252-259.

[9] K. Srinivasa Rao, R. Murali, S.K. Rajendra, Rainbow and strong rainbow criticalness of some standard graphs, Int. J. of Mathematics and Computer Research, 3, No 1 (2015), 829-836.

[10] K. Srinivasa Rao, R. Murali, S.K. Rajendra, Rainbow connection number in brick product graphs, Bull. of the Internat. Math. Virtual Institute, $\mathbf{8}$ (2018), 55-66.

[11] K. Srinivasa Rao, R. Murali, Rainbow connection number in brick product graphs $C(2 n, m, r)$, International J. of Math. Combin., 2 (2017), 70-83.

[12] S. Sy, R. Wijaya, Surahamat, Rainbow connection of some graphs, Applied Mathematical Science, 8 (2014), 4693-4696. 
